\title{
Learning Control Law of Mode Switching for Hypersonic Morphing Aircraft Based on Type-2 TSK Fuzzy Neural Network
}

\author{
Xin Jiao and Ju Jiang
}

\begin{abstract}
A novel learning method of control law of mode switching for hypersonic morphing aircraft, based on type-2 Takagi- Sugeno-Kang (TSK) fuzzy neural network, is proposed in this paper. The purpose of this method is to learn the control law of mode switching from a group of training data, in order to steadily and smoothly switch the winglets from retracting to stretching mode. In this method, taking into consideration the characteristics of type-2 fuzzy, we utilize an interval type-2 TSK fuzzy approach, the rules of which are learned from training data by back- propagation algorithm. Simulation results indicate that the proposed learning method of switching control law, based on type-2 TSK fuzzy neural network, can steadily and smoothly switch the winglets from retracting to stretching mode, providing a novel method for obtaining an excellent switching control law in situations with a group of training data.
\end{abstract}

Index Terms-Control law of mode switching, learning rules, interval type-2 TSK fuzzy, back-propagation algorithm, hypersonic morphing aircraft.

\section{INTRODUCTION}

Hypersonic morphing aircraft with retracting winglets always switch their flight modes while flying in a large range of space, so the control laws need to be changed correspondingly. It is, however, more difficult to steadily and smoothly control the switching processes for the aircrafts, with their characteristics of rapid time-variation, strong nonlinearity, strong coupling, and model uncertainty [1], [2]. Complex nonlinear systems cannot be controlled well using traditional methods, which increase the difficulty of switching control laws [3].

Recent papers have focused attention on this problem. Stability of switched systems has been researched and analyzed in [4]. A logic-based supervisor has been designed to manage the switching moments between two main controllers [5]. Inverse dynamics have generated the trajectory for transition from VTOL to wing-borne flight [6]. A switched linear parameter-varying system approach has been designed for the rigid longitudinal dynamics of a hypersonic aircraft to ensure tracking of the given commands

Manuscript received November 18, 2014; revised February 28, 2015. This work was supported in part by Funding of Jiangsu Innovation Program for Graduate Education under Grant CXZZ13_0170; Funding for Outstanding Doctoral Dissertation in NUAA under grant BCXJ13-06, and the Project Funded by the Fundamental Research Funds for the Central Universities.

The authors are with the Automation Department, Nanjing University of Aeronautics and Astronautics, Nanjing, 210016 China (e-mail: jiaoxin_mengqu@163.com,jiangju@nuaa.edu.cn). of the closed-loop system control [7]. An extended, bump-less switching technique has been proposed to offer optimal control for continuous- time systems of hypersonic aircraft via linear quadratic optimal control and an internal model principle [8]. A switching fault- tolerant control approach has been applied to an air-breathing hypersonic vehicle which was subjected to time-varying actuator and sensor faults [9]. The piece-wise linear Lyapunov functions have been used to investigate the stabilizing switching control laws for discrete- and continuous-time linear systems [10]. An adaptive switching learning PD control has been proposed for trajectory tracking of robot manipulators in an iterative operation mode [11].

However, in the current state of research, existing training data of switching control law has not been considered. For example, sometimes in practice, a pilot of a hypersonic aircraft performs a switching task casually, without switching the control law before doing so. As a result, we need to learn the switching control law from the training data, in order to instruct other aircraft.

Type-2 fuzzy [12]-[14] is a useful method for dealing with uncertainty problems. The switching control law is a kind of combination of two control laws, the coefficients of which can be regarded as Interval type-2 TSK fuzzy rules learned from training data. Hence, we study a novel learning method in this paper, based on type-2 TSK fuzzy neural network from the training data, to learn the control law of mode switching when the winglets change from retracting to stretching mode. Furthermore, the back-propagation algorithm is applied to optimize the type-2 TSK fuzzy rules so that the learned switching control law approximates the training data as much as possible. Simulation results testified to the effectiveness of this learning method.

The outline of this paper is as follows: Section II presents the model of hypersonic aircraft. In Section III, the learning control law of mode switching from training data is proposed, based on type- 2 TSK fuzzy neural network. In order to optimize the type-2 fuzzy rule, the back-propagation algorithm is applied to approximate the training data. In Section IV, we describe an experiment conducted to testify the effects of the proposed method. Finally, conclusions are presented in Section V.

\section{SLIDing Mode CONTROL LAW OF HyPERSONIC AIRCRAFT}

In this paper, we researched a hypersonic morphing flight vehicle with retracted winglets. Compared with retracting 
winglets, stretched winglets can be used to increase the lift-drag ratio, mean aerodynamic chord, and reference area. A typical hypersonic morphing aircraft's retracting and stretching winglet modes based on X-24B configuration [15], are shown in Fig. 1.

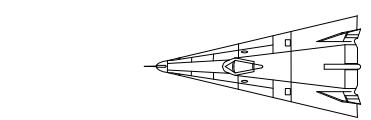

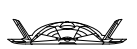

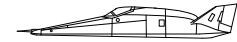

(a) The retracting mode

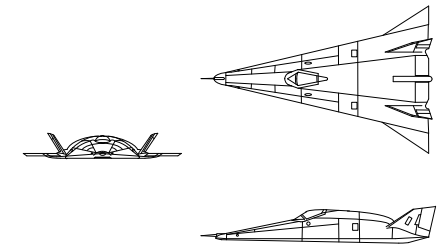

(b) The stretching mode.
Fig. 1. A typical hypersonic morphing aircraft's modes based on X-24B configuration [15].

According to the longitudinal force and moment equilibrium of hypersonic aircraft, the longitudinal model of a hypersonic flight vehicle [16] can be obtained as follows:

$$
\begin{gathered}
\dot{V}=\frac{T \cos \alpha-D}{m}-\frac{\mu \sin \gamma}{r^{2}} \\
\dot{\gamma}=\frac{L+T \sin \alpha}{m V}-\frac{\left(\mu-V^{2} r\right) \cos \gamma}{V r^{2}} \\
\dot{q}=\frac{M_{y}}{I_{y}} \\
\dot{\alpha}=q-\dot{\gamma} \\
\dot{h}=V \sin \gamma \\
\ddot{\beta}=-2 \xi \omega \dot{\beta}-\omega^{2} \beta+\omega^{2} \beta_{c}
\end{gathered}
$$

In this model, $V, \gamma, q, \alpha$, and $h$ are the aircraft's velocity, flight path angle, pitch rate, angle of attack, and altitude, respectively; $\beta, \omega$, and $\xi$ are the throttle setting, natural frequency, and damping coefficient; and $m, \mu, r$, $M_{y}$, and $I_{y}$ are the mass, gravitational constant, radial distance from the earth's center, pitching moment, and moment of inertia. $m=136820 \mathrm{~kg}, I_{y}=9490740 \mathrm{~kg} \cdot \mathrm{s}^{2}$. For the lift $L$, drag $D$, thrust $T$, we have:

$$
\begin{gathered}
L=\frac{1}{2} \rho V^{2} s C_{L} \\
D=\frac{1}{2} \rho V^{2} s C_{D} \\
T=\frac{1}{2} \rho V^{2} s C_{T} \\
C_{M}\left(\delta_{e}\right)=0.0292\left(\delta_{e}-\alpha\right) \\
M_{y}=0.5 \rho V^{2} s \bar{c}\left[C_{m}+C_{M}\left(\delta_{e}\right)\right]
\end{gathered}
$$

$$
C_{T}= \begin{cases}0.02576 \beta & \text { if } \beta \leq 1 \\ 0.0224+0.00336 \beta & \text { if } \beta>1\end{cases}
$$

where $C_{L}, C_{D}, C_{T}$, and $C_{m}$ are lift, drag, thrust, and pitch moment coefficients, respectively; and $\rho, s$, and $R_{e}$ are the density of the air, reference area, and radius of the earth.

In the retracting mode, $s=369 m^{2}, \bar{c}=27 m$, the aerodynamic parameters of lift, drag, and pitch moment coefficients for different Mach and angle of attack values are shown in Fig. 2.
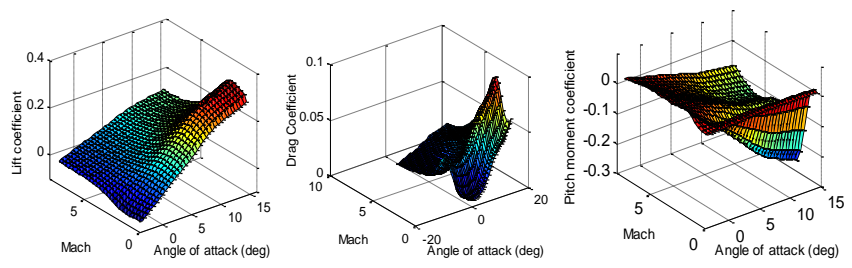

Fig. 2. The aerodynamic parameters in retracting mode.

In the stretching mode, $s=389 m^{2}, \bar{c}=30 m$, the aerodynamic parameters of lift, drag, and pitch moment coefficient for different Mach and angle of attack values are shown in Fig. 3.
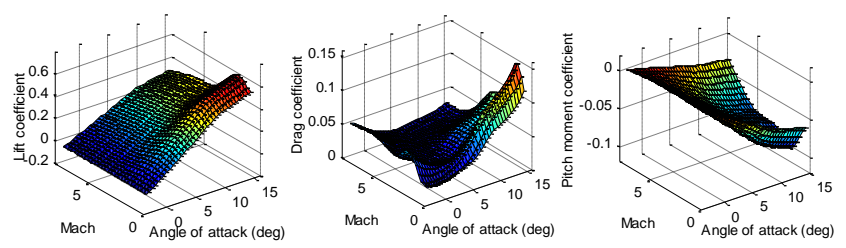

Fig. 3. The aerodynamic parameters in stretching mode.

Before time $t_{1}$, the aircraft is in retracting mode. Then, within the time interval $\left[t_{1}, t_{2}\right]$, we morph the winglets of aircraft, so that the winglets completely switch to stretching mode after time $t_{2}$.

Assumption: $s, \bar{c}$, and all the aerodynamic parameters are switched according to the following format:

$$
X(t)=X_{1}(t) e^{\left(-a\left(t-t_{1}\right)\right)}+X_{2}(t)\left(1-e^{\left(-a\left(t-t_{1}\right)\right)}\right)
$$

which $X_{1}$ stands for value in retracting mode, $X_{2}$ stands for value in stretching mode, and $X$ stands for value in switching mode. When $t=t_{1}, s, \bar{c}$, and all the aerodynamic parameters are the same as the values in retracting mode. When $t_{1}<t \leq t_{2}, s, \bar{c}$, and the weight of values in retracting mode decreases gradually, while the weight of values in stretching mode increases gradually. So, $s, \bar{c}$, and all the aerodynamic parameters are the values increasingly in stretching mode. When $t>t_{2}, s, \bar{c}$, and all the aerodynamic parameters are approximately equal to the values in stretching mode.

\section{LEARNING Method OF SWITCHING CONTROL LAW}

Define $U_{1}$ as the control law of retracting mode, and $U_{2}$ as the control law of stretching mode. When the hypersonic 
morphing aircraft switches the mode of the winglets, the control law must be switched correspondingly. The control law of the switching process is as follows:

$$
U= \begin{cases}U_{1} & t<t_{1} \\ U_{t} & t_{1}<t<t_{2} \\ U_{2} & t>t_{2}\end{cases}
$$

where $U_{t}$ is the control law of mode switching, expressed as follows:

$$
U_{t}=a U_{1}+b U_{2}+c
$$

The system structure of learning control law of mode switching is shown in Fig. 4.

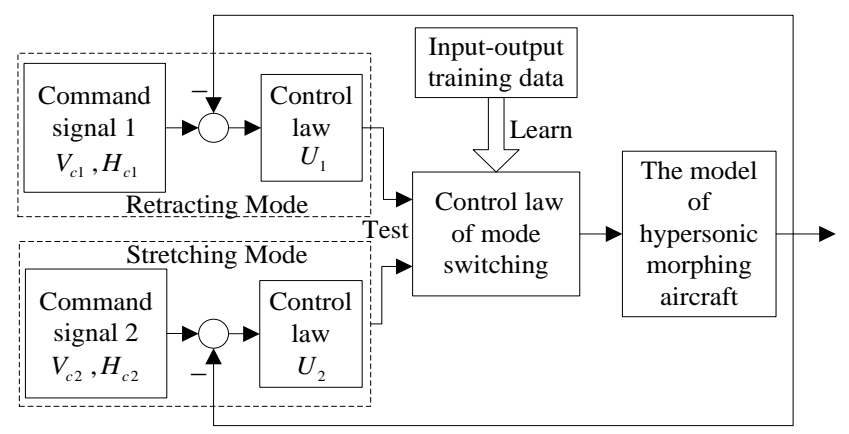

Fig. 4. The system structure of learning control law of mode switching.

In Fig. $4, U_{1}$ is the control law of retracting mode, and $U_{2}$ is the control law of stretching mode. From the input-output training data, we can learn the control law of mode switching. In order to test the performance of the learned control law, we need to examine that performance when the mode of the winglets switches from retracting to stretching mode, and compare the results with the training data.

\section{A. Interval Type-2 TSK Fuzzy}

In Fig. 4, for the group of input-output training data, there are two inputs $U_{1}, U_{2}$ and one output $U_{f}$. In a type-2 TSK fuzzy logic, IF-THEN rules are also used to describe the relationship between input and output data. We used the Gaussian primary membership function with uncertain mean as the input membership function and the output membership function. We describe the $j$-th rule of interval type-2 TSK fuzzy logic system (A2-C1 case) as:

$R^{j}:$ If $U_{1}$ is $\tilde{F}_{1}^{j}$ and $U_{2}$ is $\tilde{F}_{2}^{j}$, then $U_{f}^{j}=C_{0}^{j}+C_{1}^{j} U_{1}+C_{2}^{j} U_{2}$

where $j=1, \mathrm{~L}, M ; U_{1}$ is the control law in retracting mode and $U_{2}$ is the control law in stretching mode; $\tilde{F}_{1}^{j}$ is the interval type-2 fuzzy set of $U_{1}$, and $\tilde{F}_{2}^{j}$ is the interval type-2 fuzzy set of $U_{2} ; U_{f}$ stands for the output of type-2 TSK fuzzy; $C_{0}^{j}, C_{1}^{j}$, and $C_{2}^{j}$ are consequent parameters, which are uncertain.

For the A2-C1 case, $C_{i}^{j},(i=0,1,2)$ can be expressed as follows:

$$
C_{i}^{j}=\left[c_{i}^{j}-s_{i}^{j}, c_{i}^{j}+s_{i}^{j}\right]
$$

where $c_{i}^{j}$ denotes the mean of $C_{i}^{j}$ and $s_{i}^{j}$ denotes the spread of $C_{i}^{j}(j=1, \mathrm{~L}, M ; i=0,1,2)$.

Membership function: Suppose that $\underline{\mu}_{\tilde{F}_{i}^{j}}$ and $\bar{\mu}_{\tilde{F}_{i}^{j}}$ are the lower and upper membership functions of $\mu_{\tilde{F}_{i}^{j}}$, respectively. Then, the membership function $\mu_{\tilde{F}_{i}^{j}}$ can be expressed as

$$
\mu_{\tilde{F}_{i}^{j}}\left(U_{i}\right)=\int_{w^{j} \in\left[\mu_{\tilde{F}_{i}^{j}}\left(U_{i}\right), \bar{\mu}_{\tilde{F}_{i}^{j}}\left(U_{i}\right)\right]^{1 / w^{j}}}
$$

Gaussian primary membership function with an uncertain mean: Suppose that $\mu_{\tilde{F}_{i}^{j}}\left(U_{i}\right)$ is the Gaussian primary membership function with an uncertain mean. Then,

$$
\mu_{\tilde{F}_{i}^{j}}\left(U_{i}\right)=\exp \left[-\frac{1}{2}\left(\frac{U_{i}-m_{i}^{j}}{\sigma_{i}^{j}}\right)^{2}\right]
$$

where $m_{i}^{j} \in\left[m_{i 1}^{j}, m_{i 2}^{j}\right], i=1,2, j=1, \mathrm{~L}, M$.

Thus, the membership function $\mu_{\tilde{F}_{i}^{j}}\left(U_{i}\right)$ has an upper boundary $\bar{\mu}_{\tilde{F}_{i}^{j}}\left(U_{i}\right)$ and a lower boundary $\underline{\mu}_{\tilde{F}_{i}^{j}}\left(U_{i}\right)$.

The upper membership function $\bar{\mu}_{\tilde{F}_{i}^{j}}\left(U_{i}\right)$ is:

$$
\bar{\mu}_{\tilde{F}_{i}^{j}}\left(U_{i}\right)=\left\{\begin{array}{lc}
N\left(m_{i 1}^{j}, \sigma_{i}^{j} ; U_{i}\right) & U_{i}<m_{i 1}^{j} \\
1 & m_{i 1}^{j} \leq U_{i} \leq m_{i 2}^{j} \\
N\left(m_{i 2}^{j}, \sigma_{i}^{j} ; U_{i}\right) & U_{i}>m_{i 2}^{j}
\end{array}\right.
$$

The lower membership function $\underline{\mu}_{\tilde{F}_{i}^{j}}\left(U_{i}\right)$ is

$$
\underline{\mu}_{\tilde{F}_{i}^{j}}\left(U_{i}\right)=\left\{\begin{array}{lc}
N\left(m_{i 2}^{j}, \sigma_{i}^{j} ; U_{i}\right) & U_{i} \leq \frac{m_{i 1}^{j}+m_{i 2}^{j}}{2} \\
N\left(m_{i 1}^{j}, \sigma_{i}^{j} ; U_{i}\right) & U_{i}>\frac{m_{i 1}^{j}+m_{i 2}^{j}}{2}
\end{array}\right.
$$

Consequently, the membership function $\mu_{\tilde{F}_{i}^{j}}\left(U_{i}\right)$ is

$$
\mu_{\tilde{F}_{i}^{j}}\left(U_{i}\right)=\left[\underline{\mu}_{\tilde{F}_{i}^{j}}\left(U_{i}\right), \bar{\mu}_{\tilde{F}_{i}^{j}}\left(U_{i}\right)\right] \quad i=1,2
$$

In the type-2 TSK fuzzy logic system with meet operation under product or minimum t-norm, the result of the input and antecedent operations, which are in the firing set $\prod_{i=1}^{2} \mu_{\tilde{F}_{i}^{j}}\left(U_{i}\right) \equiv F^{j}\left(U_{i}\right)$, is an interval type-1 set [17].

$$
F^{j}(U)=\left[\underline{f}^{j}(U), \bar{f}^{j}(U)\right]=\left[\underline{f}^{j}, \bar{f}^{j}\right]
$$

where

$$
\underline{f}^{j}\left(U_{i}\right)=\underline{\mu}_{\tilde{F}_{1}^{j}}\left(U_{1}\right) \times \underline{\mu}_{\tilde{F}_{2}^{j}}\left(U_{2}\right)
$$




$$
\bar{f}^{j}\left(U_{i}\right)=\bar{\mu}_{\bar{F}_{1}^{j}}\left(U_{1}\right) \times \bar{\mu}_{\bar{F}_{2}^{j}}\left(U_{2}\right)
$$

The consequent of fuzzy rule $R^{j}$ is also an interval type-1 set, $U_{f}^{j}=\left[U_{f l}^{j}, U_{f r}^{j}\right]$, where

$$
\begin{aligned}
& U_{f l}^{j}=\sum_{i=1}^{2} c_{i}^{j} U_{i}+c_{0}^{j}-\sum_{i=1}^{2}\left|U_{i}\right| s_{k}^{j}-s_{0}^{j} \\
& U_{f r}^{j}=\sum_{i=1}^{2} c_{i}^{j} U_{i}+c_{0}^{j}+\sum_{i=1}^{2}\left|U_{i}\right| s_{k}^{j}+s_{0}^{j}
\end{aligned}
$$

The following after fuzzy inference is the type reducer. We used the center-of sets type reducer [17]

$$
\begin{aligned}
U_{f \cos }= & {\left[U_{f f}, U_{f r}\right] } \\
= & \int_{U_{f}^{1} \in\left[U_{f}^{1}, U_{f r}^{1}\right]} \mathrm{L} \int_{U_{f}^{M} \in\left[U_{n}^{M}, U_{f r}^{M}\right]} \int_{f^{1} \in\left[f^{1}, \bar{f}^{\prime}\right]} \mathrm{L} \\
& \int_{f^{M} \in\left[f^{M}, \bar{f}^{M}\right]}\left(1 / \frac{\sum_{j=1}^{M} f^{j} U_{f}^{j}}{\sum_{j=1}^{M} f^{j}}\right)
\end{aligned}
$$

where $U_{f \cos }$ is the interval set; $U_{f l}$ is the left-most point of $U_{f}$; and $U_{f r}$ is the right-most point of $U_{f}$. Then, we have:

$$
\begin{gathered}
U_{f l}=\frac{\sum_{j=1}^{M} f_{l}^{j} U_{f l}^{j}}{\sum_{j=1}^{M} f_{l}^{j}} \\
U_{f r}=\frac{\sum_{j=1}^{M} f_{r}^{j} U_{f r}^{j}}{\sum_{j=1}^{M} f_{r}^{j}}
\end{gathered}
$$

where $f_{l}^{j}$ is the left-most point of $f^{j}, f_{r}^{j}$ is the right-most point of $f^{j}, U_{f l}^{j}$ is the left-most point of $U_{f}^{j}$, and $U_{f r}^{j}$ is the right-most point of $U_{f}^{j}$.

Note that $\left[U_{f l}^{j}, U_{f r}^{j}\right],(j=1, \mathrm{~L}, M)$ must be computed before the computation of $U_{f \text { cos }}$.

To compute $U_{f l}$, we need to determine $f_{l}^{j},(j=1, \mathrm{~L}, M)$, and to compute $U_{f r}$ we need to determine $f_{r}^{j},(j=1, \mathrm{~L}, M)$. The computation procedure for $U_{f r}$ is provided briefly here.

Without loss of generality, assume that the pre-computed $U_{f r}^{j} \quad$ are arranged in ascending order; i.e., $U_{f r}^{1} \leq U_{f r}^{2} \leq \mathrm{L} \leq U_{f r}^{M}$. Then:

1) Compute $U_{f r}$ in (29) by initially setting $f_{r}^{j}=\left(\underline{f}^{j}+\bar{f}^{j}\right) / 2$ for $j=1, \mathrm{~L}, M$, where $\underline{f}^{j}$ and $\bar{f}^{j}$ have been preciously computed using (23)-(24), and let
$U_{f r}{ }^{\prime} \equiv U_{f r}$.

2) Find $R(1 \leq R \leq M-1)$, such that $U_{f r}^{R} \leq U_{f r}{ }^{\prime} \leq U_{f r}^{R+1}$.

3) Compute $U_{f r}$ in (29) with $f_{r}^{j}=\underline{f}^{j}$ for $j \leq R$, and $f_{r}^{j}=\bar{f}^{j}$ for $j>R$, and let $U_{f r}{ }^{\prime \prime} \equiv U_{f r}$.

4) If $U_{f r}{ }^{\prime \prime} \neq U_{f r}{ }^{\prime}$, proceed to Step 5. If $U_{f r}{ }^{\prime \prime}=U_{f r}{ }^{\prime}$, stop, and set $U_{f r}^{\prime \prime}=U_{f r}{ }^{\prime}$.

5) Set $U_{f r}{ }^{\prime}$ equal to $U_{f r}{ }^{\prime \prime}$, and return to Step 2.

Observe that in this algorithm, the number $R$ plays an important role. For $j \leq R, f_{r}^{j}=f^{j}$, whereas for $j>R$, $f_{r}^{j}=\bar{f}^{j}$; hence, $U_{f r}$ in (29) can be represented as

$$
\begin{aligned}
U_{f r}= & U_{f r}\left(\underline{f}^{1}, \mathrm{~L}, \underline{f}^{R}, \bar{f}^{R+1}, \mathrm{~L}, \bar{f}^{M}, U_{f r}^{1}, \mathrm{~L}, U_{f r}^{M}\right) \\
= & \frac{\sum_{j=1}^{R} \underline{f}^{j} U_{f r}^{j}+\sum_{j=R+1}^{M} \bar{f}^{j} U_{f r}^{j}}{\sum_{j=1}^{R} \underline{f}^{j}+\sum_{j=R+1}^{M} \bar{f}^{j}}
\end{aligned}
$$

The procedure for computing $U_{f l}$ is very similar to the one outlined above for $U_{f r}$. In Step 2, find $L(1 \leq L \leq M-1)$ such that $U_{f l}^{L} \leq U_{f l}{ }^{\prime} \leq U_{f l}^{L+1}$. Additionally, in Step 3, compute $U_{f l}$ with $f_{l}^{j}=\bar{f}^{j}$ for $j \leq L$ and $f_{l}^{j}=\underline{f}^{j}$ for $j>L$. Then, $U_{f l}$ in (28) can be represented as

$$
\begin{aligned}
& U_{f l}= U_{f l}\left(\underline{f}^{1}, \mathrm{~L}, \underline{f}^{L}, \bar{f}^{L+1}, \mathrm{~L}, \bar{f}^{M}, U_{f l}^{1}, \mathrm{~L}, U_{f l}^{M}\right) \\
& \sum_{j=1}^{L} \bar{f}^{j} U_{f l}^{j}+\sum_{j=L+1}^{M} \underline{f}^{j} U_{f l}^{j} \\
& \sum_{j=1}^{L} \bar{f}^{j}+\sum_{j=L+1}^{M} \underline{f}^{j}
\end{aligned}
$$

where $L$ and $R$ are the number of points which separate $U_{f l}$ and $U_{f r}$ into two sides, respectively, one side using lower firing strengths, and the other using upper firing strengths.

Consequently, the defuzzified crisp output $U_{f}$ is

$$
U_{f}=\left(U_{f l}+U_{f r}\right) / 2
$$

\section{B. Learning The Type-2 TSK Fuzzy Rules From Training Data Using a Back-Propagation Algorithm}

Given the $\mathrm{N}$ input-output training data $\left(U_{i}^{(k)}, U_{t}^{(k)}\right), i=1,2 ; k=1, \cdots, N$.

$$
J=\frac{1}{2}\left[U_{f}\left(U_{i}^{(k)}\right)-U_{t}^{(k)}\right]^{2} \quad k=1, \mathrm{~L}, N
$$

The purpose is to learn the parameters $C_{i}^{j}$ of fuzzy rules to minimize $J$ in (33). The steps to learn the type-2 TSK fuzzy rules are as outlined below: 
Step 1: Initialize all of the parameters of antecedent and consequent membership function.

Step 2: Set all the counters.

Step 3: Compute the output of type-2 TSK fuzzy according to the input data of pair-wise training data.

Compute $U_{f l}^{j}\left(U_{i}^{(k)}\right)$ and $U_{f r}^{j}\left(U_{i}^{(k)}\right)$;

Compute $\underline{f}^{j}, \bar{f}^{j}$

Compute the defuzzified output $U_{f}\left(U_{i}^{(k)}\right)$

Step 4: Update the $c_{i}^{j}$ and $s_{i}^{j}$

$$
\begin{aligned}
J\left(c_{i}^{j}, s_{i}^{j}\right) & =\frac{1}{2}\left[U_{f}\left(U_{i}^{(k)}\right)-U_{t}^{(k)}\right]^{2} \quad k=1, \mathrm{~L}, N \\
\frac{\partial}{\partial c_{i}^{j}} J\left(c_{i}^{j}, s_{i}^{j}\right) & =\frac{\partial}{\partial c_{i}^{j}}\left(\frac{1}{2}\left[U_{f}\left(U_{i}^{(k)}\right)-U_{t}^{(k)}\right]^{2}\right) \\
& =\left(U_{f}\left(U_{i}^{(k)}\right)-U_{t}^{(k)}\right) \frac{\partial}{\partial c_{i}^{j}}\left(U_{f}\left(U_{i}^{(k)}\right)\right)
\end{aligned}
$$

Update:

$$
\begin{gathered}
c_{0}^{j}: c_{0}^{j}-\alpha\left(U_{f}\left(U_{i}^{(k)}\right)-U_{t}^{(k)}\right) \frac{\left(f_{r}^{j}+f_{l}^{j}\right)}{2} \\
s_{0}^{j}: s_{0}^{j}-\alpha\left(U_{f}\left(U_{i}^{(k)}\right)-U_{t}^{(k)}\right) \frac{\left(f_{r}^{j}-f_{l}^{j}\right)}{2} \\
c_{i}^{j}: c_{i}^{j}-\alpha\left(U_{f}\left(U_{i}^{(k)}\right)-U_{t}^{(k)}\right) \frac{\left(f_{r}^{j}+f_{l}^{j}\right)}{2} U_{i}^{(k)} \\
s_{i}^{j}: s_{i}^{j}-\alpha\left(U_{f}\left(U_{i}^{(k)}\right)-U_{t}^{(k)}\right) \frac{\left(f_{r}^{j}-f_{l}^{j}\right)}{2} U_{i}^{(k)}
\end{gathered}
$$

where $i=1,2, \alpha$ is learning rate.

Step 5: Repeat until convergence.

\section{EXPERIMENTS}

We set the switching time as $35 \mathrm{~s}$. Before time $35 \mathrm{~s}$, the aircraft was in retracting mode. Then, within the time interval [35s, 40s], we morphed the winglets of aircraft, so that the winglets completely switched to stretching mode after time in 40s.

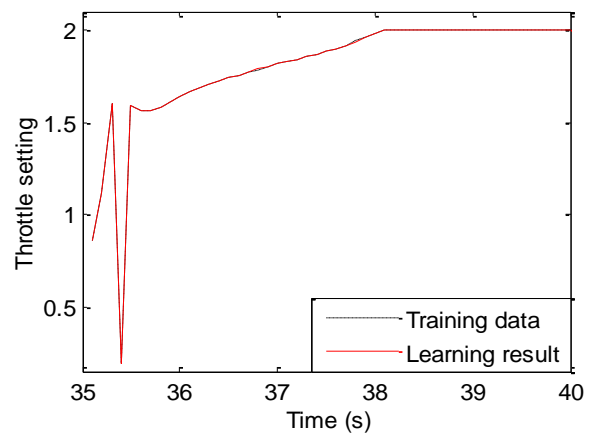

Fig. 5. Learning effect of throttle setting.
In order to better learn the control law of mode switching and overcome over training, we used $50 \%$ of the sample data for training. The learning effects of control laws in [35s, 40s] including throttle setting $\beta_{c}$ and elevator deflection $\delta_{e}$, are shown in Fig. 5 and Fig. 6.

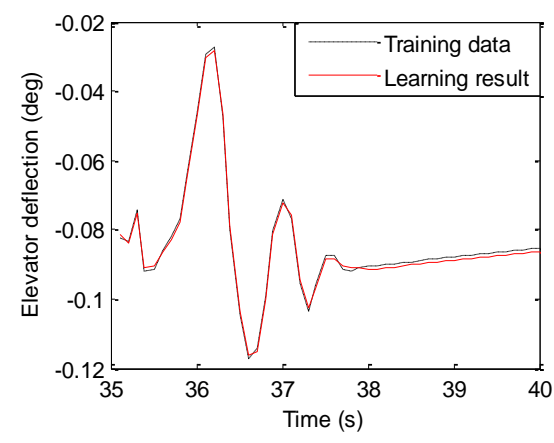

Fig. 6. Learning effect of elevator deflection.

From Fig. 5 and Fig. 6, we can see that the learned $\beta_{c}$ and $\delta_{e}$ were fairly approximate to the original training data. $\beta_{c}$, in particular, nearly overlapped with the training data. In order to test the control effects of the learned control law of mode switching, we applied the learned control law in the switching process of hypersonic morphing aircraft. Then, we obtained the switching effects of the learned control law of mode switching, as shown in Fig. 7.

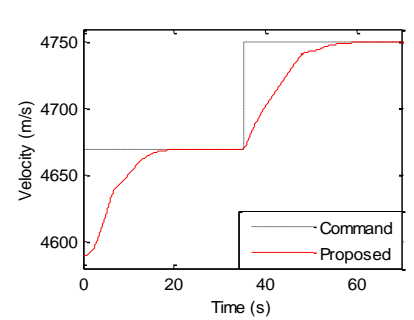

(a) Velocity

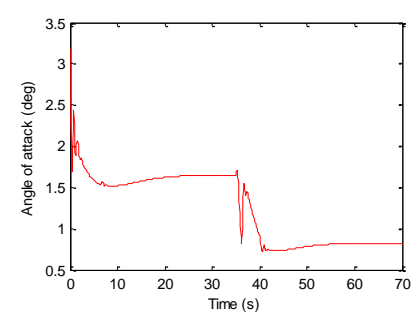

(c) Angle of attack

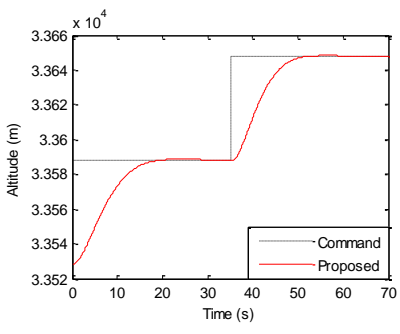

(b) Altitude

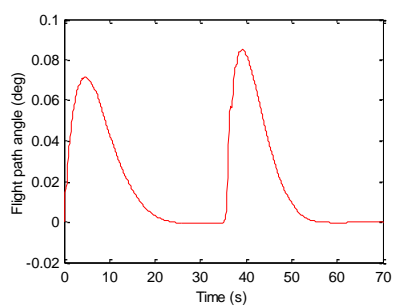

(d) Flight path angle
Fig. 7. The switching effects of learn control law of mode switching.

From Fig. 7, we can see that the before time 35s, the control law of the retracting mode could keep the hypersonic aircraft in retracting mode with good tracking performance. After time 40s, the hypersonic aircraft in stretching mode could precisely track the ideal command under the control law of stretching mode. Particularly, in the switching process within the time interval [35s, 40s], the flight states of hypersonic aircraft, including velocity, altitude, angle of attack, and flight path angle, switched very steadily and smoothly, without any saltation. The simulation results above illustrate the effectiveness of proposed learning method of control law of mode switching from retracting to stretching mode. 


\section{CONCLUSION}

The learning method of type-2 TSK fuzzy neural network has been studied in this paper in order to learn the unknown control law of mode switching for hypersonic morphing aircraft from a group of training data. When a hypersonic morphing aircraft switches the mode of the winglets from retracting to stretching mode, considering the control law of mode switching is a kind of combination of two control laws, the coefficients of the combination can be regarded as the type-2 TSK fuzzy rules. Afterwards, the back- propagation algorithm is utilized so that the learned switching control law approximates the training data as much as possible. The effectiveness of the novel learning control law of mode switching is illustrated by comparisons of learned control effects with the training data through simulation studies. The numerical simulation results indicated that the control law of mode switching learned from training data during the process of switching from retracing mode to stretching mode can keep the switching process stable and smooth.

\section{REFERENCES}

[1] Q. Wang and R. F. Stengel, "Robust nonlinear control of a hypersonic aircraft," Journal of Guidance, Control, and Dynamics, vol. 23, no. 4, pp. 577-585, July-August 2000

[2] H. J. Xu, M. D. Mirmirani, and P. A. Ioannou, "Adaptive sliding mode control design for a hypersonic flight vehicle," J Guidance, Control, and Dynamics, vol. 27, no. 5, pp. 829-838, September-October 2004.

[3] A. Ataei and Q. Wang, "Non-linear control of an uncertain hypersonic aircraft model using robust sum-of-squares method," IET Control Theory \& Applications, vol. 6, no. 2, pp. 203-215, January 2012.

[4] H. Lin and P. J. Antsaklis, "Stability and stabilizability of switched linear systems: a survey of recent results," IEEE Transactions on Automatic Control, vol. 54, no. 2, pp. 308-322, February 2009.

[5] V. M. Lapierre, E. Gagnon, A. Desbiens, and F. Wong, "Flight mode transitions for a fixed wing mini aerial vehicle from/to hovering," AIAA paper 2011-6402, 2011.

[6] A. Verma and J. L. Junkins, "Trajectory generation for transition from VTOL to wing-borne flight using inverse dynamics," AIAA paper 2000-0971, 2000.

[7] Q. Lu, L. Zhang, P. Shi, and H. R. Karimi, "Control design for a hypersonic aircraft using a switched linear parameter-varying system approach," in Proc. the Institution of Mechanical Engineers, Part IJournal of System and Control Engineering, vol. 227, no. 1, pp. 85-95, January 2013.

[8] W. Bao, Y. Qi, D. Yu, Z. Yao, and J. Zhao, "Bumpless switching scheme design and its application to hypersonic vehicle model," International Journal of Innovative Computing, Information and Control, vol. 8, no. 1B, pp. 677-689, January 2012.

[9] F. Wu and X. Cai, "Switching fault-tolerant control of a flexible air-breathing hypersonic vehicle," The Institution of Mechanical
Engineers Part I, Journal of Systems and Control Engineer, vol. 227 no. 1, pp. 24-38, January 2013.

[10] X. D. Koutsoukos and P. J. Antsaklis, "Design of stabilizing switching control laws for discrete- and continuous-time linear systems using piecewise-linear Lyapunov functions," International Journal of Control, vol. 75, no. 12, pp. 932-945, August 2002.

[11] P. R. Ouyang, W. J. Zhang, and M. M. Gupta, "An adaptive switching learning control method for trajectory tracking of robot manipulators," Mechatronics, vol. 2006, no. 16, pp. 51-61, February 2006.

[12] Q. Ren, M. Balazinski, and L. Baron, “Type-2 TSK fuzzy logic system and its type-1 counterpart," International Journal of Computer Applications, vol. 20, no. 6, pp. 8-13, April 2011.

[13] G. Zheng, J. Wang, and L. Jiang, "Research on type-2 TSK fuzzy logic systems," Fuzzy Information and Engineering, vol. 2, no. 62, pp. 491-500, 2009.

[14] M. Biglarbegian, W. W. Melek, and M. J. Mendel, "On the stability of interval type-2 TSK fuzzy logic control systems," IEEE Transactions on Systems, Man, and Cybernetics- Part B: Cybernetics, vol. 40, no. 3 , pp. 798-818, June 2010

[15] E. J. Saltzman, K. C. Wang, and W. K. Iliff, "In-flight subsonic lift and drag characteristics unique to blunt-based lifting reentry vehicles," AIAA Journal of Spacecraft and Rockets, vol. 44, no. 2, pp. 299-309, March-April 2007.

[16] J. D. Shaughnessy, S. Z. Pinckney, J. D. Mcminn, C. I. Cruz, and M. L. Kelley, "Hypersonic Vehicle Simulation Model: Winged-cone Configuration (Technical Report)," NASA Langley Research Center 1990.

[17] M. J. Mendel, Uncertain Rule-Based Fuzzy Logic Systems: Introduction and New Directions, USA: Prentice Hall PTR, 2001.

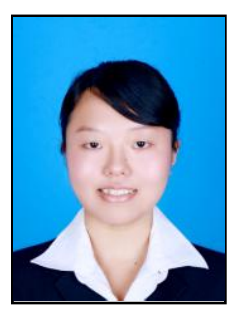

Xin Jiao received the B.S. degree in automation from Southwest University of Science and Technology, Mianyang, China, and the M.S. degree in control theory and control engineering from Nanjing University of Aeronautics and Astronautics, Nanjing, China. She is currently working toward the Ph.D degree in navigation, guidance and control in the Department of Automation, Nanjing University of Aeronautics and Astronautics, Nanjing, China.

Her current research interests include nonlinear control of hypersonic aircraft and control law of mode switching.

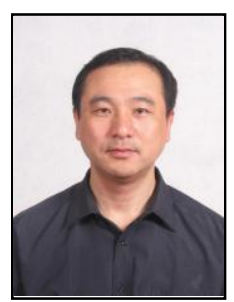

Ju Jiang received the B.S. degree in flight vehicle control and the M.S. degree in navigation, guidance and control from Beihang University, Beijing, China, and received the Ph.D. degree in machine learning and artificial intelligence in the Center for Pattern Analysis and Machine Intelligence, University of Waterloo, Waterloo, Canada.

$\mathrm{He}$ is currently the vice-president of Graduate School of Nanjing University of Aeronautics and Astronautics. His current research interests include automatic control, flight vehicle control and machine learning. Currently, Prof. Jiang is a member of IEEE. 